\title{
In Vitro Tensile Properties, Hydrolytic and Oxidative Permeability of the Biomedical Thermoplastic Polyurethane (TPU) / Organohectorite Nanocomposites
}

\author{
Azlin Fazlina Osman*1 and Darren Martin² \\ ${ }^{1}$ School of Materials Engineering, Malaysia \\ ${ }^{2}$ Australian Institute for Bioengineering and Nanotechnology, Australia
}

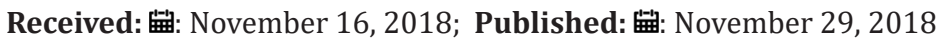

*Corresponding author: Azlin Fazlina Osman, School of Materials Engineering, 01000 Kangar, Perlis, Malaysia

\begin{abstract}
In this work, poly(dimethylsiloxane) (PDMS) / poly (hexamethylene oxide) (PHMO)-based thermoplastic polyurethane (TPU) nanocomposites incorporating nano-organo-hectorite were studied for biomedical application. This involved the investigation on the effects of oxidative and hydrolytic exposure on the performance of the host TPU and TPU-organohectorite nanocomposites through in vitro tensile and permeability tests (in water and hydrogen peroxide $\left(\mathrm{H}_{2} \mathrm{O}_{2}\right)$. Results demonstrated that the presence of nanosized organically modified hectorite (nano-organohectorite), either in single or dual modification system affects the in vitro tensile properties, hydrolytic and oxidant permeability of this TPU. Specifically, the TPU incorporating with the most hydrophobic nanofiller (organohectorite surface modified with $75 \%$ dimethyldioctadegylamminium chloride (DODMAC) / 25\% choline) exhibits the largest increment in the modulus of elasticity, retention in water and hydrogen peroxide $\left(\mathrm{H}_{2} \mathrm{O}_{2}\right)$ permeability upon four weeks of exposure at $37^{\circ} \mathrm{C}$ due to enhanced compatibility between the nanofiller and the hydrophobic PDMS soft segments.
\end{abstract}

Keywords: Thermoplastic Polyurethane; Nano-Organofluoromica, Nano-Organohectorite; Nanocomposites; In Vitro Mechanical Properties; Permeability

Abbreviations: PDMS: Poly Dimethylsiloxane; PHMO: Poly Hexamethylene Oxide; TPU: Thermoplastic Polyurethane; DODMAC: Dimethyldioctadegylamminium Chloride; BDO: 1,4 Butanediol; MDI: 4,4'-Methylene Diphenyl Diisocyanate; ODTMA: Octadecyltrimethyl Ammonium

\section{Introduction}

Polyurethanes are potential materials for biomedical applications due to their exceptional mechanical properties and biocompatibility Xiao \& Chen [1]. However, when TPUs were used alone without nanofiller addition, they commonly failed to achieve the required mechanical and physical performance as biomaterial Osman \& Osman et al. [2,3]. The addition of nanoscale inorganic particles into the TPU will improve the mechanical, physical and properties and biostability of the host material, further improving its quality as biomaterial. The poly (dimethylsiloxane) (PDMS) / poly (hexamethylene oxide) (PHMO)-based TPUs represent potential candidates for biomedical application due to their improved mechanical properties and biostability over polyether based TPUs Andriani et al. [4]. However, to develop the PDMS/ PHMO-based TPU for biomedical applications, it is crucial to ensure that its mechanical properties and biostability meet the requirements of a biomedical device. Previous study showed that the ambient mechanical properties of the PDMS/PHMO based TPU were successfully improved by incorporating the organically modified organohectorite Osman et al. [3] Thus, this project intends to further investigate the potential of PDMS/PHMO based TPU nanocomposites for biomedical applications by assessing their in vitro mechanical performance and biostability.

\section{Experimental Method}

The polymer matrix used is commercially known as ElastEon ${ }^{\mathrm{TM}}$ E5-325 TPU consists of a $1000 \mathrm{~g} / \mathrm{mol}$ poly(dimethylsiloxane) (PDMS) and 700g/mol poly (hexamethylene oxide) (PHMO) mixed soft segment in a 98:2 (w/w) ratio, and a hard segment composed of alternating 4,4'-methylene diphenyl diisocyanate (MDI) and 1,4 butanediol (BDO) sequences. The hard segment concentrations are $32.5 \mathrm{wt} \%$. The synthetic hectorite that used 
as nanofiller in the TPU was supplied by Kobo Products, Inc. Its average platelet length is approximately 30 to $50 \mathrm{~nm}$. Four types of surface modifier were used to surface modified the hectorite: Ethoquad 0/12, choline chloride, octadecyl trimethyl ammonium (bromide) (ODTMA) and dimethyldioctadecylammonium chloride (DODMAC). The PDMS/PHMO based TPU nanocomposites containing $2 \mathrm{wt} \%$ organohectorite were prepared by using solvent casting method. The procedures were explained elsewhere Osman \& Osman et al. [2,3]. (Table 1) summarizes the formulation of all the nanocomposite samples.

Table 1: Formulation of PDMS/PHMO based nanocomposite samples.

\begin{tabular}{|c|c|c|}
\hline & Formulation of Sample & Acronym \\
\hline 1 & TPU (neat) & Host \\
\hline 2 & TPU+2wt\% hectorite & $2 \mathrm{He}$ \\
\hline 3 & $\begin{array}{c}\text { TPU+2wt\% hectorite modified with 100\% } \\
\text { Ethoquad }\end{array}$ & $2 \mathrm{HeE}$ \\
\hline 4 & $\begin{array}{c}\text { TPU+2wt } \% \text { hectorite modified with 75\% } \\
\text { Ethoquad and 25\% Choline chloride }\end{array}$ & $2 \mathrm{HeE}-\mathrm{C}$ \\
\hline 5 & $\begin{array}{c}\text { TPU+2wt\% hectorite modified with 75\% } \\
\text { octadecyl trimethyl ammonium (bromide) } \\
\text { ODMTA and 25\% Choline chloride }\end{array}$ & $2 \mathrm{HeO}-\mathrm{C}$ \\
\hline 6 & $\begin{array}{c}\text { TPU+2wt\% hectorite modified with 75\% } \\
\text { dimethyldioctadecylammonium chloride } \\
\text { (DODMAC) \& 25\% Choline chloride }\end{array}$ & $2 \mathrm{HeD}-\mathrm{C}$ \\
\hline
\end{tabular}

\section{In Vitro Tensile Test}

The preliminary studies on the in vitro mechanical properties was carried out as a first step towards predicting the potential improvements in long-term in vivo performance of the TPU nanocomposites for long term implant applications. The test samples were conditioned in the environmental chamber containing $\mathrm{H}_{2} \mathrm{O}_{2}$ solution for 4 weeks before proceeding to the tensile test. The tensile test was performed according to ASTM D638 method with ten replicates of dumbbell sample from each material. Mean values of tensile strength, Young's modulus and elongation at break were taken for comparison between materials.

\section{Hydrolytic Permeability Test (in PBS Solution)}

Hydrolytic permeability test using PBS solution was done to study the hydration characteristic of the studied materials and relate it with their barrier properties. Rectangular test samples with a dimension of $\mathrm{L}=\mathrm{W}=3 \mathrm{~cm}$ was cut, oven dried for 1 hour at 50 ${ }^{\circ} \mathrm{C}$ to remove the moisture and immediately weighed to determine the initial mass. Next, the samples were immersed in the beaker containing phosphate buffered saline PBS solution and conditioned at $37{ }^{\circ} \mathrm{C}$, which approximates human body temperature. The sample mass was recorded after every 7 days of immersion (until 4 weeks' time). The hydration characteristic of each material was determined from the percent increase in mass after each weeks of immersion, using the following calculation:

Increase in mass $(\%)=($ Hydrated mass - Initial mass $) \times 100 /$ Initial mass

\section{Oxidative Permeability Test (in $\mathrm{H}_{2} \mathrm{O}_{2}$ Solution)}

Oxidative permeability test using $\mathrm{H}_{2} \mathrm{O}_{2}$ solution was done to study the barrier characteristic of the studied materials and relate it with their in vitro tensile properties. Rectangular test samples with a dimension of $\mathrm{L}=\mathrm{W}=3 \mathrm{~cm}$ was cut, oven dried for 1 hour at 50 ${ }^{\circ} \mathrm{C}$ to remove the moisture and immediately weighed to determine the initial mass. Next, the samples were immersed in the beaker containing hydrogen peroxide $\left(\mathrm{H}_{2} \mathrm{O}_{2}\right)$ solution and conditioned at $37{ }^{\circ} \mathrm{C}$, which approximates human body temperature. The sample mass was recorded after every 7 days of immersion (until 4 weeks' time). The oxidative permeability of each material was determined from the percent increase in mass after four weeks of immersion, using the following calculation:

Increase in mass $(\%)=($ Final mass - Initial mass $) \times 100 /$ Initial mass

\section{Results and Discussion}

\section{In vitro Tensile Properties of Host TPU and TPU Nanocomposites}

Tensile testing was carried on the host TPU and nanocomposites upon 4 weeks of $\mathrm{H}_{2} \mathrm{O}_{2}$ exposure to investigate the effect of oxidative exposure on the mechanical properties of these materials. Figures 1-3 compare the average values of modulus of elasticity, tensile strength, and break elongation of samples after four weeks of this oxidative treatment. Among all the samples, $2 \mathrm{HeD}-\mathrm{C}$ nanocomposite shows the highest value of modulus of elasticity. The neat TPU showed the lower value for modulus of elasticity but higher value for elongation at break as compared to the TPU nanocomposites. This shows that the incorporation of the nanofillers impart in increasing the stiffness and rigidity of the TPU matrix. Good nanofiller-TPU interactions would bring stiffening effect to the host TPU molecular chains Rao [5]. Furthermore, the incorporation of the modified hectorite into the TPU structure resulted in the lowering of its tensile strength, therefore it is suggested that the presence of organic surfactant brings catalytic effect for degradation of the TPU upon oxidative exposure.

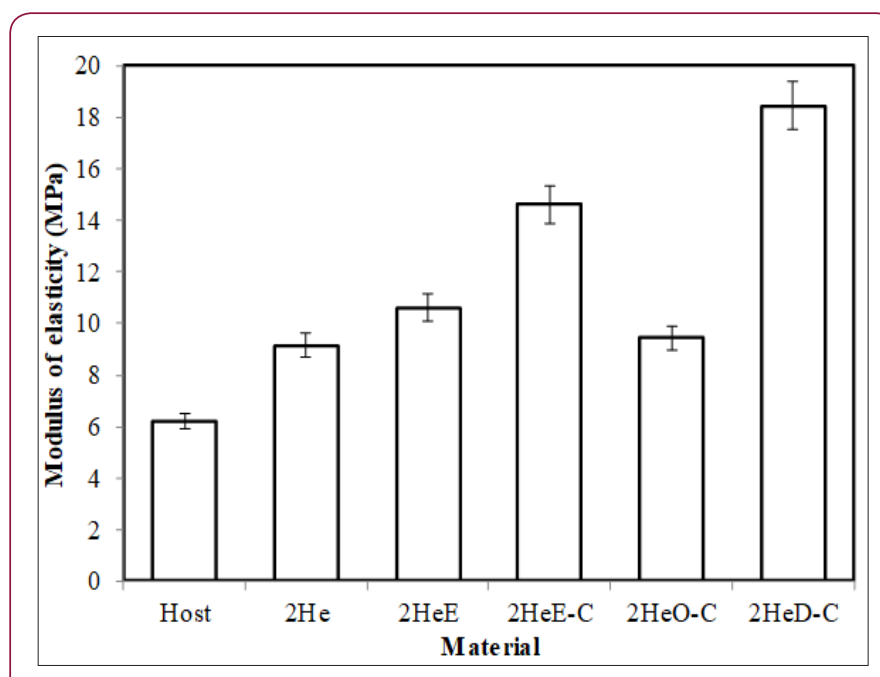

Figure 1: The modulus of elasticity of host TPU, $2 \mathrm{He}, 2 \mathrm{HeE}$, 2HeE-C, 2HeO-C and 2HeD-C, 2HeD-C nanocomposites. 


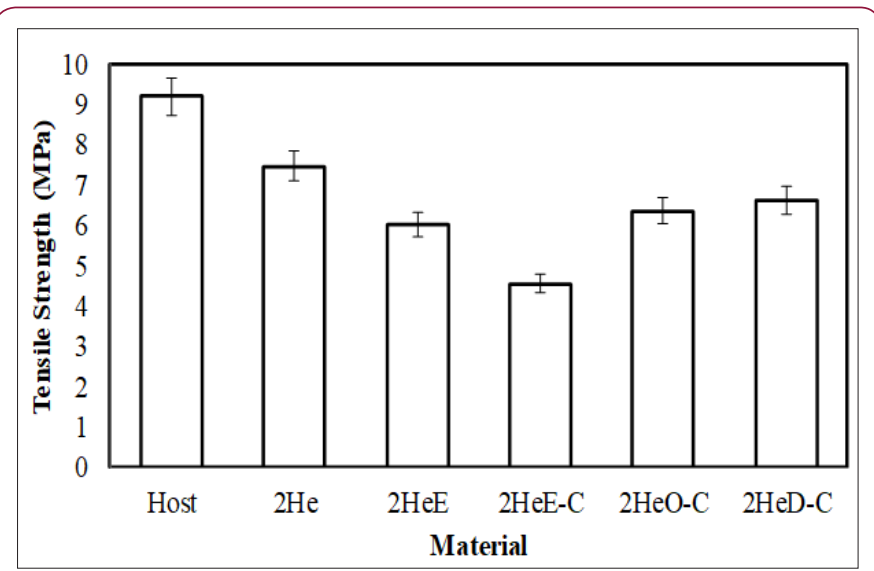

Figure 2: The tensile strength of host TPU, $2 \mathrm{He}, 2 \mathrm{HeE}$ 2HeE-C, 2HeO-C and 2HeD-C, 2HeD-C nanocomposites.

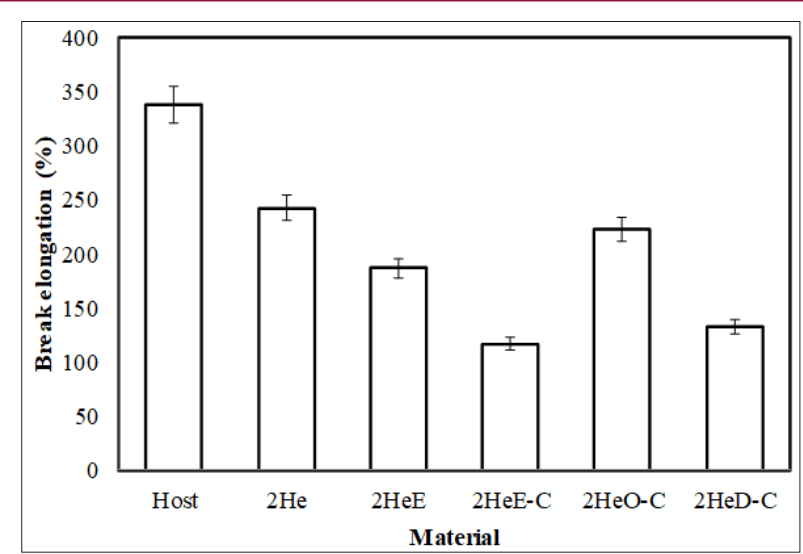

Figure 3: The elongation at break of host, $2 \mathrm{He}, 2 \mathrm{HeE}$, $2 \mathrm{HeE}-\mathrm{C}, 2 \mathrm{HeO}-\mathrm{C}$ and $2 \mathrm{HeD}-\mathrm{C}, 2 \mathrm{HeD}-\mathrm{C}$ nanocomposites.

However, with the presence of the hydrophobic dual modifier (HeD-C) on the hectorite surface, the modulus of the TPU increased to a larger extent. This could be probably due to enhanced compatibility between the most hydrophobic organohectorite and the TPU matrix system that contains hydrophobic PDMS soft segment. As a result, the molecular motions of soft segments were greatly restricted. According to the previous work (Andriani), the hectorite with dual surface modification (HeE-C) is more hydrophobic than its single modification counterpart (HeE). Enhanced hydrophobicity of the hectorite through dual surface modification allows more preferential interactions between the hydrophobic soft segments and the organohectorite nanofiller, thereby it induces more restricted soft segment mobility Osman \& Osman et al. [2,6,7].

\section{Hydrolytic Permeability of the TPU/Organohectorite Nanocomposites}

Changes in the weight of the neat TPU and TPU nanocomposites before and after exposed in PBS solution at $37^{\circ} \mathrm{C}$ for four weeks were observed to determine the permeability of the materials. The increase of mass is assumed as a result of water permeability inside the TPU structure. From the data obtained as shown in (Figure 4), the water permeability of host TPU sample was the same level as the 2HeD-C nanocomposite but lower compared to other samples. This means that the water permeability ability of TPU was retained after the incorporation of $2 \mathrm{wt} \% \mathrm{HeD}-\mathrm{C}$ nanofiller but increased with the addition of other types of nanofiller. This might be due to $2 \mathrm{HeD}-\mathrm{C}$ organohectorite with dual modifications (DODMAC/CC) possesses the most hydrophobic characteristic, since the DODMAC has two long alkyl chains Osman, Andriani et al. $[3,8]$. On the other hand, $2 \mathrm{HeE}$ showed the highest water uptake in PBS solutions. This might be due to Ethoquad has the hydroxyl $(\mathrm{OH})$ group in the structure, therefore it possesses the least hydrophobic characteristic Osman, Andriani et al. [3,8]. From the result, $2 \mathrm{HeE}-\mathrm{C}$ shows the second highest increased in mass percentage.

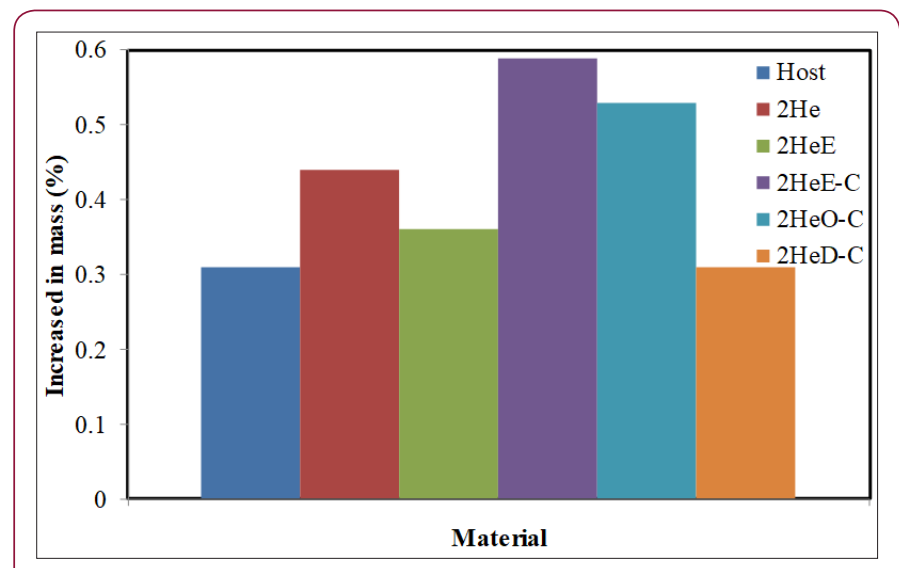

Figure 4: Increase in mass percentage after 4 weeks of PBS $\left(37^{\circ} \mathrm{C}\right)$ exposure.

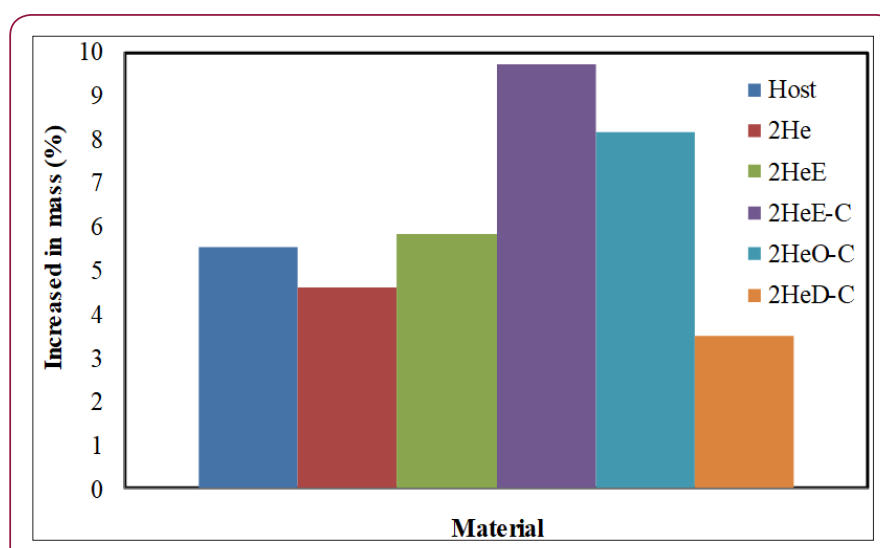

Figure 5: Increase in mass percentage after 4 weeks of $\mathrm{H}_{2} \mathrm{O}_{2}\left(37^{\circ} \mathrm{C}\right)$ exposure.

This might also due to the present of $\mathrm{OH}$ group in the Choline chloride structure. Oxidative Permeability of the TPU/ Organohectorite Nanocomposites (Figure 5) shows the effect of oxidative agent on the TPU nanocomposites. As stated by Heath, Cooper and Lawrie [9], most of the TPU materials are prone to oxidation especially the one contains polyether in the soft segments. The impermeability towards oxidative agent may hinder the oxidative degradation process in the TPU. In this experiment, the increase of mass is assumed as a result of oxidative permeability inside the TPU structure. The TPU incorporated with HeD-C shows the lowest increased in mass upon $\mathrm{H}_{2} \mathrm{O}_{2}$ exposure. This might due to the presence of more compatible organosilicate as nanofiller in the TPU matrix, which contribute to greater bonding and interface 
interactions between the nanofiller and the TPU matrix, thereby resulting in more tortuous path for the entering of the oxidants.

\section{Conclusion}

The PDMS/PHMO-based TPU nanocomposite incorporating organohectorite with dual surface modification system (most hydrophobic characteristic) has better in vitro mechanical properties than the TPU nanocomposite incorporating organohectorite with single modification. This could be associated with enhanced compatibility between the nanofiller and the host TPU as the TPU contains the hydrophobic siloxane chains in its soft segment structure. This led to enhanced matrix barrier towards oxidative and hydrolytic permeability.

\section{Acknowledgement}

The authors thank Aortech Biomaterials Pty Ltd for research materials and Miss Ho Yeek Ming for sample testing.

\section{References}

1. Xiao J, Chen S (2016) Tensile properties of medical grade thermoplastic polyurethane. Polymeric Materials Science and Engineering 32(1,9): 4548.

2. Osman AF (2013) Biomedical Thermoplastic Polyurethane Nanocomposite: Structure-Properties Relationship. PhD Thesis, University of Queensland, Australia.

\section{ISSN: 2574-1241}

DOI: $10.26717 / B J S T R .2018 .11 .002120$

Azlin Fazlina Osman Biomed J Sci \& Tech Res

(C) (i) This work is licensed under Creative (c) Commons Attribution 4.0 License

Submission Link: https://biomedres.us/submit-manuscript.php
3. Osman AF, Halley P, Martin D (2013) Impact of Controlled Hydrophobicity of the Organically Modified Silicates on the Properties of Biomedical Thermoplastic Polyurethane (TPU) Nanocomposites. Advanced Materials Research 795: 9.

4. Andriani Y, Morrow IC, Taran E, Edwards GA, Schiller TL, et al. (2013) In vitro biostability of poly (dimethyl siloxane/hexamethylene oxide)based polyurethane/layered silicate nanocomposites. Acta Biomaterialia 9(9): 8308-8317.

5. Rao Y (2017) Engineering of Interface in Nanocomposites Based on PU Polymers in Polyurethane Polymers: Composites and Nanocomposites, Elsevier Inc: 73-133.

6. Osman AF, Andriani Y, Edwards GA, Schiller TL, Jack KS, et al. (2012) Engineered nanofillers: impact on the morphology and properties of biomedical thermoplastic polyurethane nanocomposites. RSC Advances 24: 9151-9164.

7. Osman AF, Edwards GA, Schiller TL, Andriani Y, Jack KS, et al. (2012) Structure-Property Relationships in Biomedical Thermoplastic Polyurethane Nanocomposite. Macromolecules 45(1): 198-210.

8. Andriani Y, Jack KS, Gilbert EP, Edwards GA, Schiller TL, et al. (2013) Organization of mixed dimethyldioctadecylammonium and choline modifiers on the surface of synthetic hectorite. Journal of Colloid and Interface Science 409: 72-79.

9. Heath D E, Cooper SL, Lawrie WG (2012) Polyurethane, in Biomedical Science: An Introduction to Materials in Medicine. In Buddy D, Ratner BD, Allan S Hoffman, Frederick J Schoen, Jack E Lemons J E (Eds.) (3 $3^{\text {rd }}$ edn.), Elsevier, MA USA: 82.

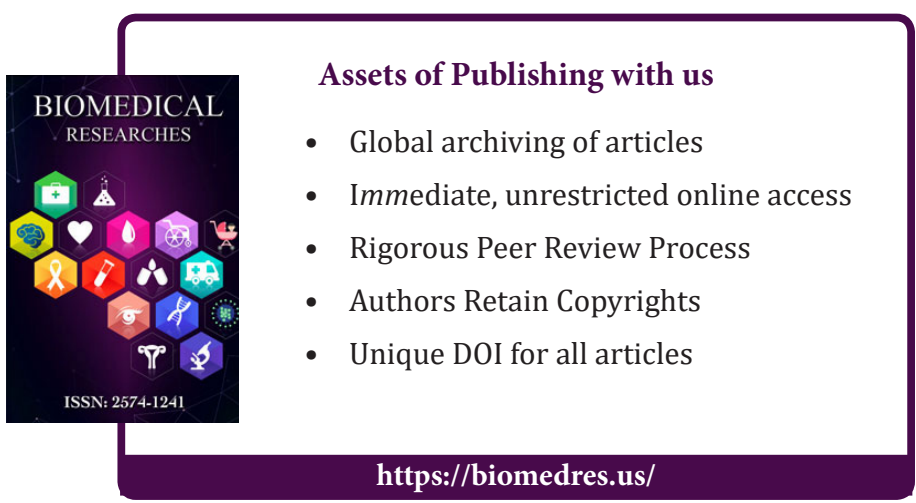

Cite this article: Azlin Fazlina 0, Darren M. In Vitro Tensile Properties, Hydrolytic and Oxidative Permeability of the Biomedical Thermoplastic Polyurethane (TPU) / Organohectorite Nanocomposites. Biomed J Sci \& Tech Res 11(4)-2018. BJSTR. MS.ID.002120. DOI: 10.26717/ 